\title{
LA PLANIFICACIÓN TURÍSTICA EN ESPACIOS NATURALES PROTEGIDOS
}

\author{
POR \\ ALFREDO TOLÓN BECERRA Y ANDRÉS GARCÍA LORCA
}

\section{Introducción}

El principal objetivo de esta investigación es diseñar un modelo de desarrollo turístico en los Espacios Naturales Protegidos, integrado en la planificación propia del Espacio, teniendo presente nuestra situación en Europa y la repercusión de la Cumbre de Río de Janeiro.

Las definiciones de Espacio Natural Protegido han madurado con el tiempo y actualmente son numerosas y variadas, pero en la mayoría de ellas se aprecian dos características a destacar: los méritos de protección y el carácter efectivo de la protección.

En primer lugar, para que un Espacio Natural sea protegido, debe ser previamente protegible, es decir, merecedor de ser protegido, lo que significa que debe reunir una serie de requisitos y cualidades extraordinarias.

El segundo elemento característico hace referencia a la efectividad de esa protección. Para que un Espacio Natural sea efectivamente protegido, deben utilizarse instrumentos eficaces.

La finalidad de un Espacio Natural Protegido ha ido evolucionando desde sus orígenes hasta constituir actualmente una institución que abarca un amplio conjunto de funciones.

Las finalidades de los primeros Espacios Naturales Protegidos (Parque de Yellostone, 1872) eran muy reducidas y se limitaban básicamente a fun-

Alfredo Tolón Becerra. Departamento de Ingeniería Rural. Universidad de Almería.

Andrés García Lorca. Departamento de Historia, Geografía e Historia del Arte. Universidad de Almería.

Estudios Geográficos, LXIII, 247, 2002 
ciones estéticas y de protección frente a las amenazas del desarrollismo industrial y urbanístico. Más adelante, en el año 80, F. López Ramón reconducía a tres grupos los fines que persiguen los Espacios Naturales Protegidos: fines de tutela o conservación de la naturaleza, fines de goce público y fines socioeconómicos ${ }^{1}$, si bien estos últimos no eran considerados prioritarios.

Estas clasificaciones han sido superadas con el tiempo, y hoy se puede afirmar que los Espacios Naturales Protegidos cumplen un conjunto de finalidades que se pueden agrupar en cinco categorías ${ }^{2}$ : de protección y conservación del medio biofísico y cultural, científica y de investigación, educativa, recreativa y socioeconómica.

Se resumen en el Cuadro I las características actuales de un Espacio Natural Protegido.

\section{CUADRo I}

RESUMEN DE LAS CARACTERÍSTICAS

DE UN ESPACIO NATURAL PROTEGIDO

\begin{tabular}{|c|c|}
\hline Natural & $\begin{array}{l}\text { En el que la intervención del hombre no ha llegado a alterar } \\
\text { de forma significativa la presencia y funcionamiento de los } \\
\text { demás elementos, bioticos y abióticos que lo integran. }\end{array}$ \\
\hline $\begin{array}{l}\text { Protegido } \\
\text { Lo que requiere: }\end{array}$ & $\begin{array}{l}\text { - Ser merecedor de protección por sus cualidades extraor- } \\
\text { dinarias. } \\
\text { - Utilizar instrumentos eficaces para su protección efectiva. }\end{array}$ \\
\hline $\begin{array}{l}\text { Que cumple las } \\
\text { siguientes } \\
\text { Finalidades: }\end{array}$ & $\begin{array}{l}\text { - De protección y conservación del medio biofísico y cul- } \\
\text { tural. } \\
\text { - Científica y de investigación. } \\
\text { - Educativa. } \\
\text { - Recreativa. } \\
\text { - Socioeconómica. }\end{array}$ \\
\hline
\end{tabular}

Fuente: Elaboración propia (1999).

\footnotetext{
${ }^{1}$ LÓPEZ RAMÓN, F., 1980. La conservación de la naturaleza: los espacios naturales protegidos. Real Colegio de España. Bolonia. p. 97.

2 Fernández DE TejadA, A., 1994. La definición de Espacios Naturales. Revista ESTRATOS, n. ${ }^{\circ} 31$, primavera. Madrid, p. 13.
}

Estudios Geográficos, LXIII, 247, 2002 
Con el objeto de abordar el tema con perspectiva histórica, se ha repasado la evolución de los Espacios Naturales Protegidos, donde los expertos han diferenciado distintos periodos caracterizados por hechos relevantes, lo que ha supuesto que la actuación en esta materia no haya sido homogénea en el tiempo. En particular, se ha podido justificar la diferenciación en tres grandes etapas fundamentales.

La primera etapa se caracteriza por el nacimiento, organización y consolidación de los primeros Espacios Naturales Protegidos, principalmente Parques Nacionales y una concepción estática y elitista de los mismos. Las finalidades de estos espacios eran de estética y recreo, y de protección de la naturaleza virgen frente a un desarrollo exacerbado.

La segunda etapa supone el desarrollo del sistema de Espacios Naturales Protegidos, con un crecimiento espectacular en superficie y en número de declaraciones. Este desarrollo viene acompañado de una ampliación de las finalidades y de los instrumentos de gestión, y de un papel creciente de los Organismos y Disposiciones Internacionales. En el plano tipológico, se asiste a una proliferación de figuras que obliga a los Organismos a establecer clasificaciones de referencia, y se constituyen y desarrollan las primeras redes. En Europa, nace con fuerza el Parque Natural Regional o asimilado, que se convierte en la principal figura en superficie.

La tercera etapa viene marcada por los acontecimientos históricos en torno a la Cumbre de Río de Janeiro, que vincula todas las políticas al desarrollo sostenible del planeta. De esta forma, los ENP deben integrarse en la política global de conservación y desarrollo sostenible, siendo necesario, por tanto, impulsar procesos de desarrollo en los ENP y sus zonas adyacentes.

La concepción de los ENP, por tanto, ha madurado notablemente. Los principios inspiradores que regían los primeros espacios declarados y que configuraron un espíritu, que se podría denominar de Yellostone en honor al primer Parque Nacional del mundo, han dado paso a un nuevo espíritu, el de Río de Janeiro. En el Cuadro II se indica de forma sintética el contraste de ambas concepciones.

Con el objeto de contribuir a superar los nuevos retos que deben afrontar los Espacios Naturales Protegidos en esta etapa crucial, se pretende diseñar un modelo de desarrollo turístico, de acuerdo con las nuevas directrices marcadas por los acontecimientos en torno a la Cum-

Estudios Geográficos, LXIII, 247, 2002 
CuAdro II

PRINCIPIOS INSPIRADORES DE UN ENP

(YELLOSTONE Y RÍO DE JANEIRO)

\begin{tabular}{ll}
\hline Espíritu de Yellostone (1872) & Espíritu de Río de Janeiro (1992) \\
\hline Santuario Natural & Integrado en un sistema abierto \\
Protección & Conservación y desarrollo sostenible \\
Estático & Dinámico \\
Gestión centralizada & Gestión descentralizada: ámbito local \\
Regulación & Planificación Integral \\
Gestión aislada & Coordinación y cooperación \\
Elitista & Participación social \\
Reacción al desarrollo & Integración con el desarrollo \\
Objetivos contrapuestos al Desarrollo & Convergencia de objetivos: Compati- \\
& bilidad \\
Excluyente con respecto a otros sec- & Complementario \\
tores & \\
Superficie reducida y delimitada & Superficie amplia: zonas de transición \\
& o Preparques \\
\hline
\end{tabular}

bre de Río de Janeiro, para su aplicación en nuestro territorio español. Este modelo deberá tener presente:

- El contexto europeo.

- La realidad española y autonómica y, en particular, su legislación.

- Las características intrínsecas de los ENP españoles y en especial, los aspectos socioeconómicos y la planificación.

Por eso, antes de abordar el diseño del modelo de desarrollo, habrá que conocer la evolución de la planificación legal y los modelos técnicos de planificación de ENP.

\section{La planificación legal}

La construcción de la actual estructura planificadora legal de los ENP en España se ha elaborado a partir de dos fuentes. De un lado, por la propia legislación específica que, aunque de una forma muy endeble ha 
aportado ciertos elementos planificadores, y de otro, por la legislación urbanística cuya aportación ha sido inicialmente más significativa, por su mayor tradición planificadora.

La planificación legal actual de los Espacios Naturales Protegidos viene marcada por la vigente Ley $4 / 89$ y por las leyes autonómicas para aquellos Espacios cuya competencia recae en las Comunidades Autónomas. De esta forma, la Ley 4/89 establece un nuevo marco jurídico que debe ser tenido en cuenta, bien directamente, bien como referencia básica, al emprender la ordenación de tales espacios. A raíz del traspaso de competencias, cada Comunidad Autónoma ha desarrollado su propio sistema de planificación de ENP de forma que las leyes autonómicas han creado una gama diversa de planes y programas de carácter más específico. Mención especial merece la legislación andaluza que incluye específicamente los planes y programas de carácter socioeconómico.

La evolución de la planificación legal, resumida en el Cuadro III, nos permitirá, con una óptica histórica, conocer con más detalle el contenido de cada uno de los planes.

El estudio de los distintos planes se realiza de forma progresiva, comenzando por el de carácter más general, el PORN, para ir descendiendo en los de carácter más específico. A modo de conclusión, se resume en el Cuadro IV las características básicas de los distintos planes considerados en la nueva estructura planificadora de acuerdo con la legislación vigente.

En el caso de la Comunidad Andaluza, la Ley 2/89 de Espacios Naturales protegidos contempla dos figuras para los Parques Naturales, el Plan de Desarrollo Integral y los Programas de Fomento. Derivado también del PORN, el Plan de Desarrollo Integral del parque es de contenido exclusivamente económico, basado en la salvaguardia de la estabilidad ecológica medio ambiental del área. La aprobación inicial de este plan corresponde al Comité de Acciones Integradas para el Ecodesarrollo, y se aplicará mediante Programas de Fomento elaborados por el Instituto de Fomento de Andalucía, canalizando así las ayudas técnicas y financieras que la Ley prevé, y que tendrá que aprobar el Consejo de Gobierno para cada uno de los Parques Naturales ${ }^{3}$.

${ }^{3}$ Garay Zabala, J., y Molina Vázquez, F. (1991). Un modelo armónico de Gestión de Espacios Protegidos: el sistema andaluz. Prácticas para la planificación de Espacios Naturales. Castroviejo, M. (De). ICONA. MAPA. Madrid. p. 60.

Estudios Geográficos, LXIII, 247, 2002

$$
-307-
$$


Cuadro III

EVOLUCIÓN DE LA PLANIFICACIÓN LEGAL DE ENP EN ESPAÑA

\section{Legislación específica en ENP}

Reglamento de la Ley de Montes (1962)

- Plan de Protección, Conservación y Disfrute.

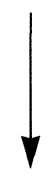

Ley de Espacios Naturales Protegidos (1975)

- Plan de Conservación, Fomento, Mejora y Disfrute

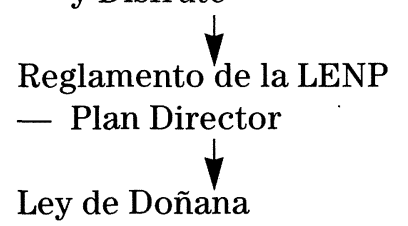

- PRUG<smiles>C[13CH3]</smiles>

\section{Legislación del suelo}

Ley del Suelo de 1956

- Planes Provinciales

- Planes Generales

- Planes Especiales

Ley del suelo de 1975

- Plan Nacional de Ordenación

- PDTC

- PGMO

- PEPMF

$$
\text { - PDTC }
$$

Ley de Parques Nacionales Canarios (1980)

- PRUG

- Planes Especiales

Ley $4 / 89$ (Estatal)

- PORN

- PRUG

- Otros planes

$\downarrow$

Leyes Autonómicas

- PORN

- PRUG

- Planes Especiales y Programas

- Planes de Carácter Socieconómico:

* PDI (L2/89 Andalucía)

* Programas de Fomento (L2/89 Andalucía)

Estudios Geográficos, LXIII, 247, 2002 
CuAdro IV

CARACTERÍSTICAS BÁSICAS DE LOS DISTINTOS PLANES DE ENP

\begin{tabular}{|c|c|}
\hline Plan & Características básicas \\
\hline PORN & $\begin{array}{l}\text { - Cabecera de grupo Normativo ( marco para los demás } \\
\text { instrumentos) } \\
\text { - Contenido integral } \\
\text { - Criterios orientadores: } \\
\text { *Ordenación de la utilización de los recursos natu- } \\
\text { rales. } \\
\text { *Ordenación territorial. } \\
\text { *Ordenación sectorial. } \\
\text { *Actividades propias del ENP. } \\
\text { - Período de vigencia o renovación: } 8 \text { años }\end{array}$ \\
\hline PRUG & $\begin{array}{l}\text { - Directrices sobre actividades propias del ENP: } \\
\text { *Uso público } \\
\text { *Investigación } \\
\text { - Período de vigencia o renovación: } 4 \text { años. }\end{array}$ \\
\hline $\begin{array}{l}\text { Planes Especiales } \\
\text { (o programas) }\end{array}$ & $\begin{array}{l}\text { - Desarrollan las directrices del PORN y del PRUG } \\
\text { - Grado de detalle suficiente para la redacción de pro- } \\
\text { yectos. } \\
\text { - Períodos de vigencia o renovación: Inferiores a } 4 \\
\text { años. }\end{array}$ \\
\hline $\begin{array}{l}\text { Planes o Programas } \\
\text { de Carácter } \\
\text { Socioeconómico }\end{array}$ & $\begin{array}{l}\text { - Por lo general adoptan la forma de planes especiales. } \\
\text { - Legislación Andaluza (L2/89) } \\
\text { *PDI Infraestructuras y equipamientos. } \\
\begin{array}{ll}* \text { PF } & \text { Fomento de actividades económicas. } \\
& \text { (Programa de desarrollo turístico) }\end{array}\end{array}$ \\
\hline
\end{tabular}

El papel de los Planes de Desarrollo Integral y Programas de Fomento es económico, y su contenido se diferencia claramente de los del PORN y PRUG ya que se trata de instrumentos que programan actuaciones concretas dentro del marco fijado por los anteriores planes: Los PDI y PF se ajustan a las orientaciones contenidas en el planeamiento anterior, y operarán como instrumentos esencialmente autónomos 
vinculados con los demás a través del proceso de seguimiento y actualización de la intervención pública en los espacios protegidos. ${ }^{4}$

A su vez, el Plan de Desarrollo Integral se diferencia de los Programas de Fomento, el Plan de Desarrollo Integral se centra en actuaciones ligadas directamente con el desarrollo económico a través de actuaciones públicas en materia de infraestructura y equipamientos, mientras que el Programa de Fomento tiene un contenido de carácter financiero y fiscal, estableciendo también medidas de asesoramiento y apoyo a empresarios y Administraciones.

Actualmente, se ha sustituido la denominación de Plan de Desarrollo Integral por la de Plan de Desarrollo Sostenible (PDS).

\section{Los modelos técnicos de planificación}

Clarificado el panorama planificador del ENP y el proceso de elaboración de planes y programas, es necesario analizar ahora las técnicas de elaboración de dichos planes, es decir, el paso del estudio del ENP a la confección del plan deseado. Para ello, se repasan los principales modelos que se han seguido en la historia de la planificación de ENP.

A pesar del carácter relativamente reciente de la planificación de $\mathrm{ENP}$, los modelos generados han sido muy variados y heterogéneos. Unos insisten en la inventariación y el análisis, otros hacen mas hincapié en el proceso de planificación y otros enfatizan el contenido de los planes. Por otro lado, es preciso puntualizar que los modelos técnicos de planificación, generalmente, se han utilizado para construir planes contemplados en la legislación que, en mayor o menor medida, fijaban directrices y criterios orientadores para su elaboración, de forma que, en algunos casos, es difícil discernir la planificación legal del modelo técnico de planificación. En cualquier caso ambos aspectos están muy relacionados, y se han repasado los modelos más significativos, con una óptica amplia, destacando sus características más relevantes. Se analizan también modelos extranjeros que han servido de fuente para modelos nacionales y que enriquecerán la base sobre la que se diseñará el modelo de desarro-

\footnotetext{
${ }^{4}$ Castanyer Vila, J., 1991. La Planificación de Espacios Protegidos: un mandato legal. Prácticas para la planificación de Espacios Naturales. Castroviejo, M. (Ed.). ICONA. MAPA. Madrid, p. 20.
}

Estudios Geográficos, LXIII, 247, 2002

$$
-310-
$$


llo socioeconómico. En la Tabla $\mathrm{V}$ se recogen los aspectos mas significativos.

Esta recopilación de las aportaciones más significativas servirá para marcar, con perspectiva histórica, los condicionantes por parte del sistema planificador del ENP para el diseño de un modelo de desarrollo turístico. A partir del análisis y conclusiones de los modelos de planificación de ENP y considerando sus aportaciones más significativas, se puede afirmar que un modelo que sirva para realizar un plan de desarrollo turístico en un ENP debe cumplir las siguientes premisas:

- El modelo debe estar integrado en la estructura planificadora del ENP.

- Debe elaborar el plan a partir del diagnóstico integral del ENP.

- Debe recoger las directrices y orientaciones marcadas en los planes existentes de carácter anterior o superior.

- Debe permitir, tras una evaluación, la modificación y mejora de planes anteriores o superiores existentes e incluso del diagnóstico en un mecanismo de retroalimentación.

- Debe tener una fuerte participación social, considerando los problemas locales desde el inicio del proceso.

- Debe considerar la realidad multiadministrativa, aplicando los principios de coordinación y cooperación.

- Debe dar una gran importancia a la figura del Equipo Planificador.

- Debe incluir estudios de capacidad e impacto con el objeto de evaluar la aptitud física de las actividades potenciales.

- Debe incorporar el conjunto de tecnologías innovadoras disponibles (SIG, telecomunicaciones...).

\section{Propuesta de modelo de planificación}

En este apartado se define un modelo para la aplicación de un proceso de desarrollo turístico en los Espacios Naturales Protegidos a partir de las experiencias en el campo del desarrollo rural y de los condicionantes propios de los ENP. En primer lugar se definen el Marco de Referencia y los Actores implicados en el modelo.

El Marco de Referencia abarca todos aquellos aspectos que deben considerarse en un proceso de planificación de desarrollo turístico, en par-

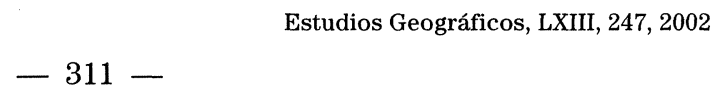




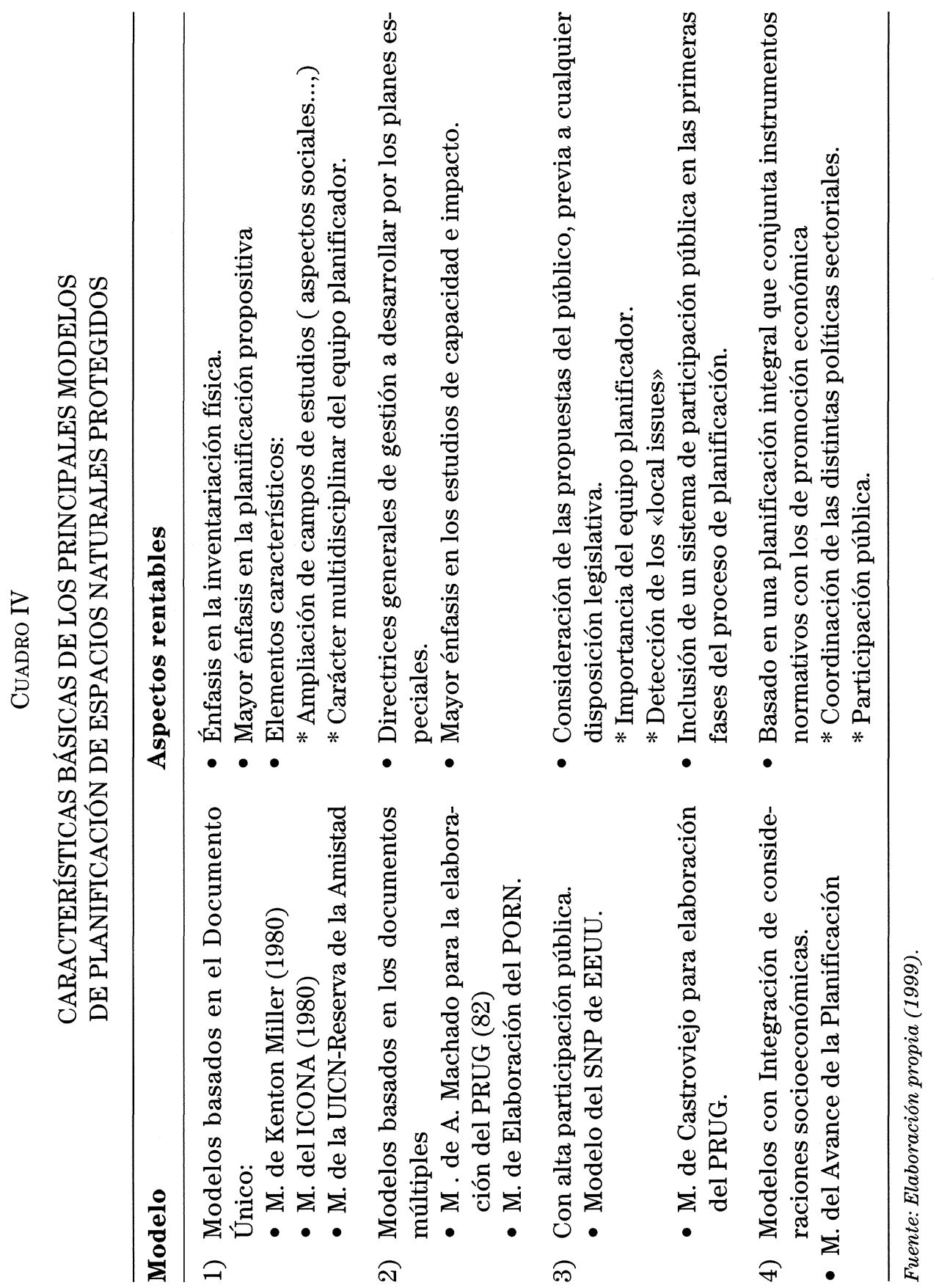

Estudios Geográficos, LXIII, 247, 2002 
ticular los aspectos conceptuales que recogen los nuevos principios inspiradores de las nuevas políticas de ENP, el marco europeo definido por sus políticas, su normativa y sus recursos institucionales, los aspectos legales y los criterios técnicos de integración en la planificación del ENP y la base ecológica de la planificación del desarrollo.

Los grupos de Actores identificados en este modelo son la Administración, la Población Local y el Equipo de Desarrollo Local-Planificador. Debe ser la Administración la que acometa un proceso de desarrollo turístico basado en el Aprendizaje Social que será protagonizado por la Población Local. Para ello es fundamental la figura del Equipo Planificador o de Desarrollo Local, encargado de la promoción directa de este desarrollo. Por eso se analizan las características generales que debe tener un Equipo de Desarrollo equilibrado, así como los instrumentos innovadores que debe utilizar, principalmente en el campo de la nuevas tecnologías y la investigación.

El desarrollo del modelo se lleva a cabo mediante un conjunto de Fases independientes pero interrelacionadas, protagonizadas por un proceso intenso de participación social, que confluyen en una última fase que supone la elaboración de unas Estrategias de Desarrollo Compatible a partir de las ideas expresadas por la población local.

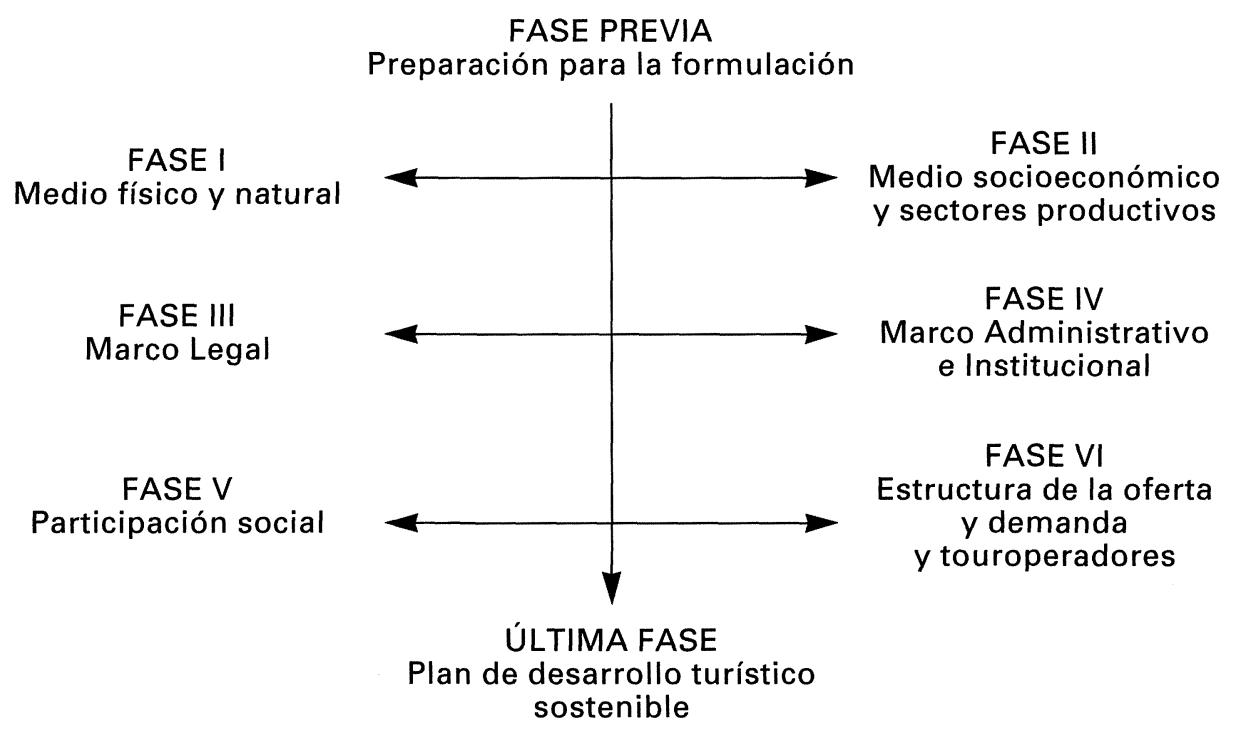

FIG. 1.-Proceso de elaboración de un Plan de Desarrollo Turístico Sostenible. 
Se recomienda realizar el proceso de participación social por etapas de forma que se comience por una primera aproximación mediante consultas con los responsables de la promoción del desarrollo, Expertos locales, Pioneros, Líderes y Alcaldes de los municipios integrantes. En etapas posteriores se mantendrán reuniones y entrevistas en profundidad con la población al objeto de conocer su opinión sobre el entorno , y recoger propuestas que sirvan de base para la definición de estrategias de desarrollo. También se pueden entrevistar a expertos locales para determinar de forma objetiva el análisis de la Capacidad e Impacto de las actividades territoriales seleccionadas.

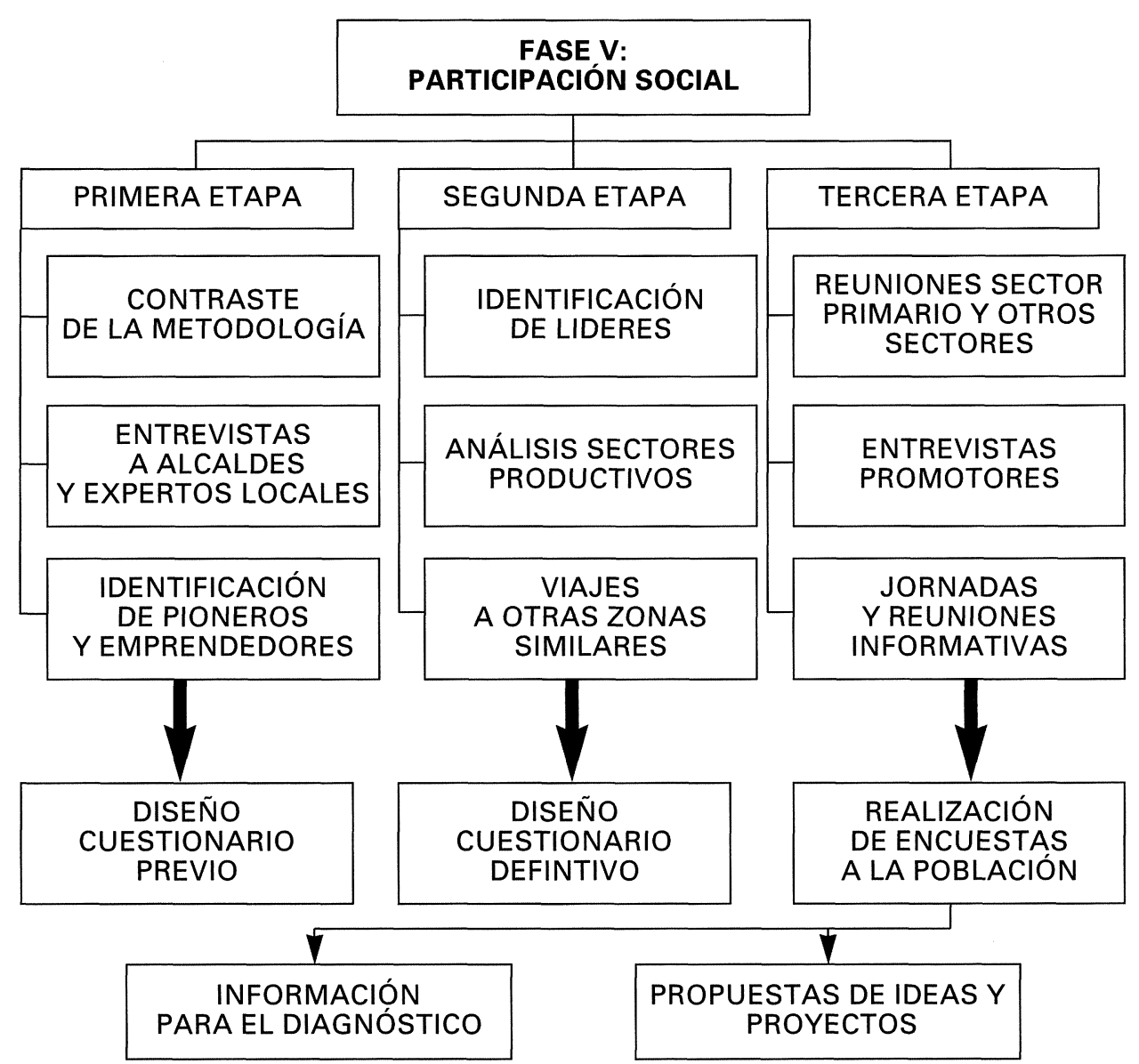

Fig. 2.-Proceso de Participación Social correspondiente a la Fase V del modelo.

Estudios Geográficos, LXIII, 247, 2002 
Las propuestas elaboradas son evaluadas de un lado por el grupo planificador y de otro, por un grupo de representantes de la Administración Ambiental. El primero las valora según el diagnóstico realizado en las cinco primeras fases y el segundo según sus propios criterios. A continuación, se seleccionan las actividades con mayor puntuación y se realiza, con la ayuda de la opinión de los expertos locales, un estudio de capacidad e impacto y posterior asignación óptima.

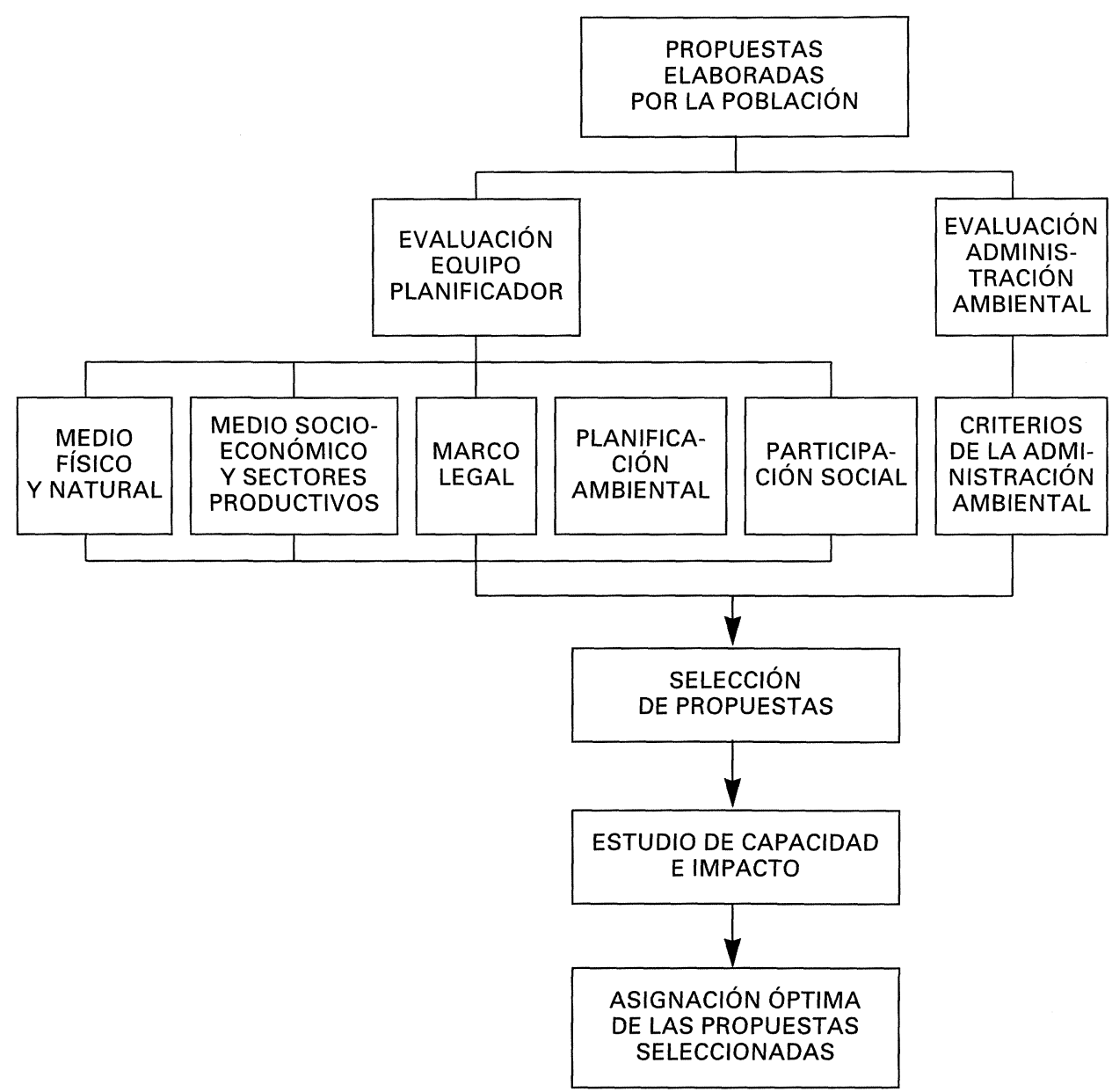

FIG. 3.-Proceso de Elaboración de Estrategias de Desarrollo correspondiente a la Fase VII del modelo de desarrollo turístico en Espacios Naturales Protegidos.

Estudios Geográficos, LXIII, 247, 2002 


\section{Aplicación práctica en un espacio natural protegido}

Con el objeto de validar la aportación en el diseño del metamodelo de planificación del desarrollo socioeconómico en ENP, se realiza una aplicación práctica en el Parque Natural de Sierra María-Los Vélez (Almería), que presentaba unas características interesantes desde el punto de vista de los objetivos de esta investigación. Ésta se inició con el estudio de los antecedentes de este Parque natural, que puso de manifiesto la existencia de una problemática social y de unas oportunidades en cuanto a legislación y sistema planificador para la puesta en marcha de un proceso de desarrollo socioeconómico.

Tras la concreción de los Términos de Referencia y de los Actores, como consecuencia de una adaptación del metamodelo, se lleva a cabo su desarrollo mediante un conjunto de fases independientes e interrelacionadas.

Estas fases, consistentes en el análisis y diagnóstico del medio físico y natural, del medio socioeconómico y los sectores productivos, del medio legal, de la planificación ambiental, de la participación social y de la administración ambiental, permiten elaborar en la última fase unas estrategias de desarrollo a partir de las propuestas de la población local.

La aplicación del modelo pone de relieve una respuesta significativa de la población al proceso de participación social iniciado y las posibilidades reales de determinadas actividades que significarían una integración de la finalidad socioeconómica con la conservación del Parque Natural.

En concreto, las actividades que abarcan mayor superficie son las de leguminosas y adehesamiento (en torno al 35\% del Parque) y las repoblaciones de protección (20\%) en zonas de mayor riesgo de erosión. El cultivo de plantas aromáticas se ha reducido a pequeñas áreas dispersas por su acción negativa sobre la erosión, dado su carácter monoespecífico. En cuanto al resto de actividades, ligadas al turismo, presentan pocos problemas de incompatibilidad, y permiten concluir que este sector será, sin duda, el que mejor contribuirá al desarrollo socioeconómico de la zona.

De esta forma, se obtienen unas Estrategias de desarrollo turístico a partir, de un lado, de un proceso de dinamización que tiene por objeto la promoción de actividades económicas, y de otro, de un control ambiental que asegura la compatibilidad de esas actividades con el medio. Este control queda garantizado con la existencia de cuatro filtros ambientales: en la valoración y selección de las propuestas según el Medio Físico y Na-

Estudios Geográficos, LXIII, 247, 2002

$$
-316-
$$


tural, según su adecuación a los Planes del ENP y según la Administración Ambiental, y en el análisis de impacto de las actividades seleccionadas. Este doble proceso permite alcanzar un equilibrio acorde con los principios del desarrollo sostenible.

\section{Conclusiones}

La potenciación de la finalidad socioeconómica en los Espacios Naturales Protegidos no debe considerarse como una amenaza sobre su estado de conservación, sino como un instrumento eficaz, si se gestiona adecuadamente, para contribuir al desarrollo sostenible del medio.

El inicio de la aplicación del modelo de desarrollo socioeconómico en el Parque Natural de Sierra María-Los Vélez ha sido, en líneas generales, positivo. La respuesta de la población local ha sido significativa y se detectan claras posibilidades de realizar actividades productivas de carácter endógeno y compatible con las finalidades del ENP. Pero será el desarrollo posterior del modelo, con la creación de la Gerencia de Promoción del Parque y la puesta en marcha de proyectos concretos, el que nos permita un seguimiento y una evaluación mas exhaustiva.

En el caso concreto del Parque Natural de Sierra María-Los Vélez, y dado que el turismo parece ser la actividad con mayores posibilidades de contribuir al desarrollo global de la zona, se propone una línea de investigación que determine las características de ese turismo. La investigación abarcaría diversos aspectos entre los que destacarían un estudio del potencial turístico proveniente de las provincias del sureste español por su buena comunicación, sin descartar otros ámbitos y una investigación social sobre la demanda y las necesidades prioritarias de los visitantes. Esta se realizaría mediante encuestas a los visitantes, durante un periodo de tiempo suficiente, y de las que se deducirán, entre otros, los siguientes aspectos:

- Clasificación de los visitantes en función de su interés principal: turismo casual, contemplador, excursionista, aventurero, montañero, cultural, científico,...

- Lugares del Parque Natural preferidos por los visitantes y motivo de la preferencia.

- Grado de sensibilidad ambiental de los visitantes.

Estudios Geográficos, LXIII, 247, 2002

$$
-317-
$$


- Valoración de los servicios ofrecidos por el Parque Natural: recreativos, de hostelería y gastronomía, de información y guía, infraestructuras..., y del personal responsable.

- Requerimientos prioritarios por parte de los visitantes, así como deficiencias observadas y sugerencias.

Los resultados obtenidos ayudarán a perfeccionar el modelo, adaptándolo a los cambios conceptuales, legislativos... que puedan producirse y a extender esta idea al resto de los ENP, principalmente de Europa.

Existen claras oportunidades para llevar a cabo procesos de desarrollo socioeconómico en estos espacios, pero para ello hacen falta nuevos modos de actuar, principalmente por parte de las personas pertenecientes a la esfera administrativa, con la ayuda de los empresarios de la planificación. La población local y la naturaleza lo agradecerán.

\section{BIBLIOGRAFÍA}

ABOAL, J. L. (1982): Análisis de la política de parques y espacios protegidos en España. Planificación y gestión de Espacios Naturales Protegidos. Fundación Conde del Valle de Salazar. Madrid.

AlieR, J. L., ATANCE, I. y De los Ríos, I. (1996): Estrategias de Desarrollo para la Sierra Oeste de Madrid. Universidad Politécnica de Madrid.

Castanyer Vila, J. (1991): La Planificación de Espacios Protegidos: un mandato legal. Prácticas para la planificación de Espacios Naturales. Castroviejo, M. (Ed.). ICONA. MAPA. Madrid.

Castroviejo Bolíbar, M. (1991): El énfasis en la participación Pública: un nuevo esquema para la redacción de los Planes Rectores. Prácticas para la Planificación de Espacios Naturales. Castroviejo, M. (Ed.). ICONA. MAPA. Madrid.

Cazorla Montero, A. (1995). La Planificación del desarrollo rural. Planificación e Ingeniería: nuevas tendencias. Cazorla, A. (Ed.). Madrid.

CNNPA de la UICN (1994): Parques para la vida: Plan de Acción para las Áreas Protegidas de Europa. ICONA.

Decreto 78/1994, de 5 de abril, por el que se aprueba el Plan de Ordenación de Recursos Naturales y el Plan Rector de Uso y Gestión del Parque Natural de Sierra María-Los Vélez. BOJA n. ${ }^{\circ} 80$, de 1 de junio de 1994.

ESPARC-95. Conclusiones. 1995. Actas del I Seminario de Espacios Naturales Protegidos. Soto del Real. Madrid.

FERnÁNDEZ DE TEJADA, A. (1994). La definición de Espacios Naturales. Revista ESTRATOS, n. ${ }^{\circ} 31$, primavera. Madrid.

Fernández, P.; PÉREz, E.; LuCIO, J. V. (1997): Criterios de definición de las categorías de protección de espacios naturales protegidos del Estado Español. Centro de Investigaciones Ambientales Fernando González Bernáldez. Serie Documentos, n. ${ }^{\circ} 23$. Comunidad de Madrid.

GaRAY ZABALA, J., y Molina VÁZquez, F. (1991): Un modelo armónico de Gestión de Espacios Protegidos: el sistema andaluz. Prácticas para la planificación de Espacios Naturales. Castroviejo, M. (De). ICONA. MAPA. Madrid.

Estudios Geográficos, LXIII, 247, 2002

$$
-318-
$$


Gómez-Limón, F. J.; MúgicA, M.; MuÑoz, C., y Delucio, J. V. (1996): Uso recreativo de los espacios naturales en Madrid. Frecuentación, Caracterización de visitantes e impactos ambientales. Serie Documentos n. ${ }^{\circ}$ 19. Centro de Investigación de Espacios Naturales Protegidos. Fernando González Bernáldez. 75 pp. ISBN84-89198-23-3.

GONZÁLEZ BERNÁLDEZ, F. (1988): Relación entre espacios naturales protegidos y protegibles. Los términos de una polémica. Supervivencia de los Espacios Naturales. Survie de Espaces Naturels. Casa de Velázquez. MAPA. Madrid.

Inventario de Espacios Naturales Protegidos de la Sección del Estado Español de la FPNNE. 1995. Lucio Férnandez, J. V. (Director). Comunidad de Madrid.

Lentisco Puche, J. D. (1995): El Parque Natural de Sierra María-Los Vélez (Almería). Revista Velezana. Ayuntamiento de Vélez Rubio (Almería).

Ley 15/1975 de 2 de mayo, de Espacios Naturales Protegidos.

Ley $2 / 1989$, de 18 de julio, por la que el Parlamento de Andalucía aprueba el inventario de Espacios Naturales Protegidos de Andalucía y se establecen medidas adicionales para su protección. Sevilla. BOJA n. ${ }^{\circ} 60$, de 27 de julio de 1989.

Ley 4/1989 de 27 de Marzo, de Conservación de las Espacios Naturales y de la Flora y Fauna Silvestres.

LÓPEZ RAMÓN, F. (1980): La conservación de la naturaleza: los espacios naturales protegidos. Real Colegio de España. Bolonia.

MORILlo FERNÁNDEZ, C., y LECONTE, J. (1988): Conclusiones generales. Supervivencia de Espacios Naturales. Survie des Espaces Naturels. Casa Velázquez. MAPA. Madrid.

MÚGICA DE LA GUERRA, M. (1993): Modelos de demanda paisajistica y uso recreativo de los espacios naturales. Tesis doctoral. Departamento de Ecología: Universidad Autónoma de Madrid.

Otero PASTOR, I. (1995): La Planificación Ambiental. Planificación e Ingeniería: nuevas tendencias. Cazorla, A. (Ed.). Madrid.

PALUZíE I MIR, L. (1990): Los espacios naturales protegibles. UPC. Barcelona.

Quintana CaVANILlaS, J. (1997): Formación Profesional de los equipos locales de Desarrollo Rural. El Ingeniero Agrónomo en el Contexto de la Nueva Política de Desarrollo Rural. DE Los Ríos, I. (Coord.). Fundación Premio Arce. Madrid.

Ramos, A. (1979): Planificación física y Ecología. Modelos y métodos. EMESA. Madrid.

RAMOS, A., y EsCRIBANO, R. (1982): Planificación y gestión: algunas consideraciones generales. Las comunidades rurales y la gestión de los Parques Naturales. Diputació de Barcelona. Servei de Parcs Naturals.

RequeJo LiBERAL, J. (1991): Los Planes de Desarrollo Integral y los Programas de Fomento de Andalucía: el proceso de elaboración. Prácticas para la Planificación de Espacios Naturales. Castroviejo, M. (Ed.). ICONA. MAPA. Madrid.

Robinson, A. (1991): La Planificación en el Sistema Nacional de Parques de los Estados Unidos. Prácticas para la Planificación de Espacios Naturales. Castroviejo, M. (Ed.). ICONA. MAPA. Madrid.

Sebastián Palomares, J. (1996): Historia, evolución y futuro de los Parques Nacionales. Conferencia en la EUIT forestal. UPM. Madrid.

RESUMEN: El presente trabajo de investigación pretende diseñar, con perspectiva histórica, un modelo de Planificación turística para Espacios Naturales Protegidos.

En primer lugar, se estudia la concepción de los ENP, que ha madurado notablemente, y su evolución histórica. Posteriormente, se estudia la evolución de la planificación legal de los ENP en España, así como la actual estructura planificadora.

Finalmente, se analizan los modelos técnicos de elaboración de planes sectoriales que, a pesar del carácter relativamente reciente de la planificación de ENP, han sido muy 
variados y heterogéneos y permiten definir los aspectos metodológicos para la obtención final de un Plan de Desarrollo Turístico compatible.

Este se construirá a partir de las ideas propuestas por la población local, y deberá integrarse en la estructura planificadora del ENP.

Palabras Claves: Espacio Natural Protegido, Planificación, Turismo sostenible, Participación local.

ABSTRACT: The present work of investigation tries to design, with a historical perspective, a model of tourist Planning for Protected Natural Spaces.

In the first place, the conception of the PNS, which has matured remarkably, is studied as well as its historical evolution. Later, the evolution of the legal planning of the PNS in Spain, and the present planning structure are also studied.

Finally, the technical models of processing of sectorial plans are analysed and, in spite of the relatively recent character of the PNS planning, these models have been very varied and heterogenous and allow to define the methodologic aspects for the final obtaining of a Plan of compatible Tourist Development.

This plan will be constructed from the proposed ideas by the local population and will have to be integrated in the planning structure of the PNS.

KEYwORDS: Protected Natural Spaces, Planning, Sustanaible Tourism, Local Participation.

RESUMÉ: Le travail actuel de recherche a l'intention de concevoir, dans une perspective historique, un modèle de la planification du tourisme pour les Espaces Naturels Protégés.

Premierement, on étudie la conception de l'ENP, qui a notablement mûri, ainsi que son évolution historique. On procède ensuite a une étude de l'évolution de la planification légale des ENP en Espagne et de la structure actuelle de planification.

Finalement, on analyse les modèles techniques du traitement des plans sectoriels qui, malgré le caractère relativement récent de la planification des ENP, ont été très divers et hétérogènes, et permettent de définir les aspects méthodologiques pour l'obtention finale d'un Plan du Développement Touristiqee compatible.

Ce plan sera construit a partir des idées proposées par la population locale, et devra être intégré dans la structure de planification de l'ENP.

Mots-CLÉS: Espaces Naturels Protégés, Planification, Tourisme Sustentable, Participation Locale.

Estudios Geográficos, LXIII, 247, 2002

$$
-320-
$$

